\title{
Synthesis and characterization of the samples
}

\section{Synthesis of poly(thiophene-3- methyl acetate) (P3TMA)}

The poly(thiophene-3-acetic acid) (P3TAA) is obtained through saponification of the ester groups of P3TMA chains, first synthesized by an oxidative-coupling polymerization, 29,30 as proposed by Kim et al. ${ }^{27}$ The 3 TAA monomer was purchased from Acros and first esterified into 3 TMA. ${ }^{27}$ Some modifications to the original procedure were found to increase the reproducibility of the synthesis and helped us to obtain large quantities of relatively high molecular weight P3TMA:

a. Anhydrous $\mathrm{FeCl}_{3}$ was conditioned under Ar atmosphere in a glove box in a set of individual vials that were then sealed under vacuum.

b. Gaseous $\mathrm{HCl}$ produced during the oxidative-coupling reaction was removed by bubbling continuously Ar through the reacting medium.

c. After several trials, no influence of the polymerization temperature on the regioregularity could be detected between $0^{\circ} \mathrm{C}$ and room temperature. Therefore it was kept at $\mathrm{T}=15^{\circ} \mathrm{C}$ for the final synthesis.

d. A typical reaction in our final conditions involved the drop-by-drop addition of pure 3TMA $(\approx 0.012 \mathrm{~mol})$ into distilled chloroform $(100 \mathrm{~mL})$ containing $\mathrm{FeCl}_{3}(\approx 0.07 \mathrm{~mol})$ with a molar ratio $\left[\mathrm{FeCl}_{3}\right] /[\mathrm{TMA}]=5.93 \pm 0.70$. In these conditions, we found that the consumption of the monomers and the evolution of molecular weights distribution came to a level in about 2 hours. This conclusion was reached by taking small aliquots about $1 \mathrm{~mL}$ from the reacting medium. These aliquots were then diluted in $20 \mathrm{~mL}$ of chloroform, and then washed with $20 \mathrm{~mL}$ of distilled water. The organic phase was separated, dried over $\mathrm{MgSO}_{4}$, and evaporated after filtration. The resulting solid phase was then redissolved in tetrahydrofuran (THF) and characterized by size exclusion chromatography (SEC) as described below.

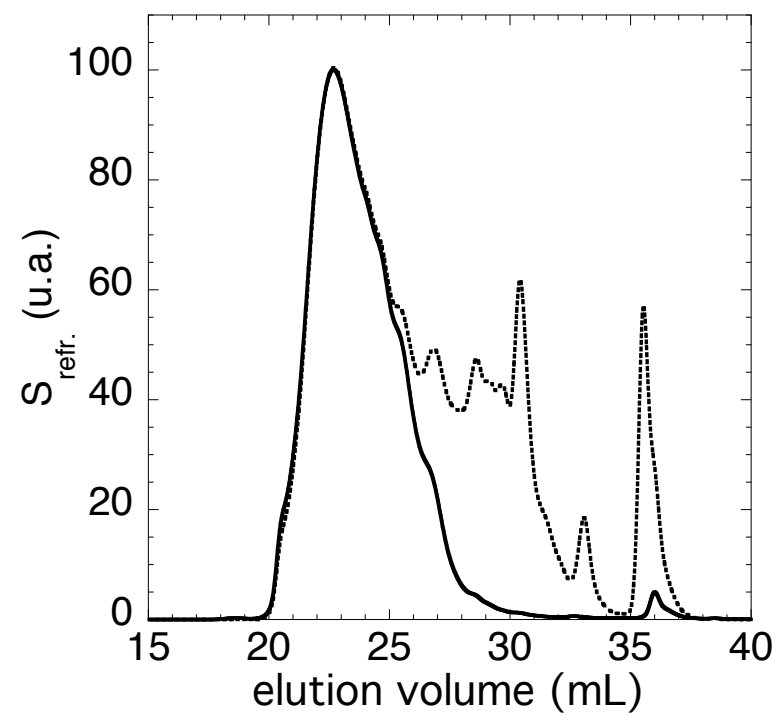

Figure 1. Size exclusion chromatograms for P3TMA after the polymerization (dotted line) and after 
elimination of the oligomers by precipitation into methanol (continuous line).

Thus after 6 hours, the reacting medium was washed several times with distilled water to eliminate the iron salts. About $25 \mathrm{~g}$ of 3 TMA were polymerized under these conditions in 13 runs. SEC analysis was performed on aliquots taken from the washed reacting medium and diluted in THF. The SEC chromatograms appeared very similar for all the runs and revealed several separated peaks at the low molecular weight side (Fig. 1, dotted line). These oligomers were eliminated by precipitation in methanol. It is worth noticing that precipitation was favoured after the addition of a few drops of diluted $\mathrm{HCl}$. The polymer was then dried under vacuum. We checked by SEC measurements in THF that all these samples had similar size distributions and mixed them in THF to obtain a large batch sample that was again precipitated in methanol. This precipitate $(9.3 \mathrm{~g}$ ) was then fractionated by Soxhlet extraction, successively by different solvents to obtain fractions with increasing molecular weights: first 12 hours by methanol, then 1 hour by acetone and finally 2 hours by acetone. For each step, both the soluble and the solid polymer parts were characterized by SEC, NMR spectrocopy and UV-visible spectroscopy. The yield of precipitated polymer was about $35 \%$ with the procedure described above.

\section{Characterization of P3TMA}

SEC. Size exclusion chromatography used THF as eluent (flow rate: $1 \mathrm{~mL} / \mathrm{min}$ ) through five Polymer Laboratory gel columns (mixed B, $30 \mathrm{~cm}$ ). For most of the comparative analysis, only apparent molecular weights deduced from calibration by polystyrene standards were determined (Table 1). However for the lowest and highest molecular weight fractions, light scattering (DAWN) coupled on line with SEC provided us with a more accurate estimation of the molecular weight and also with a calibration curve for P3TMA eluted in THF.

\begin{tabular}{|c|c|c|c|c|c|c|}
\hline \multicolumn{3}{|c|}{ SEC } & \multicolumn{4}{c|}{${ }^{13}$ C NMR doublet } \\
\cline { 1 - 2 } PS calibration & P3TMA calibration & \multicolumn{4}{c|}{ relative area fraction } \\
\cline { 1 - 2 }$M_{\mathrm{w}}$ & $M_{\mathrm{w}} / \mathrm{M}_{\mathrm{n}}$ & $M_{\mathrm{w}}$ & $130.0 \mathrm{PPm}$ & $128.8 \mathrm{PPm}$ & $127.4 \mathrm{PPm}$ & $126.2 \mathrm{PPm}$ \\
\hline 5800 & 1.5 & 10000 & 0.26 & 0.31 & 0.22 & 0.21 \\
\hline 7000 & 1.5 & 11900 & 0.29 & 0.36 & 0.19 & 0.16 \\
\hline 24000 & 2.2 & 41000 & 0.33 & 0.28 & 0.24 & 0.15 \\
\hline
\end{tabular}

Table 1. Characteristics of the P3TMA fractions.

NMR spectroscopy. $\quad{ }^{1} \mathrm{H}$ and ${ }^{13} \mathrm{C}$ NMR spectroscopies were performed on a Bruker AC40O spectrometer in $\mathrm{CDCl}_{3}$ solvent. The use of a $\pi / 4$ pulse angle eliminated the signal from quaternary carbons so that the signal from the protonated carbon in the thiophene ring can be easily measured. As expected for non-regioregular P3TMA four peaks appear corresponding to the four possible tryads (Table 1). Moreover no significant difference in the 
regioregularity of the different fractions could be detected.

UV-visible absorption. Absorption spectra were measured with a Varian Cary 50 Scan spectrophotometer in THF solvent in the range $300<\lambda(\mathrm{nm})<800$ of incident wavelength $\lambda$. Here we comment only on the position $\lambda_{\max }$ of the absorption maximum. It can be noticed that $\lambda_{\max }$ increases with the molecular weight of the chains up to $\lambda_{\max }=403 \pm 2 \mathrm{~nm}$ for molecular weights larger than $13000 \mathrm{~g} / \mathrm{mol}$ (polythiophene calibration) (Fig. 2). Polydispersity effects explain the large degree of polymerization ( $D P_{n} \approx 80$ ) apparently needed to reach the plateau value of $\lambda_{\max }$. It is to be noticed that the data in Fig. 2 correspond to different batch samples fractionated following the same method.

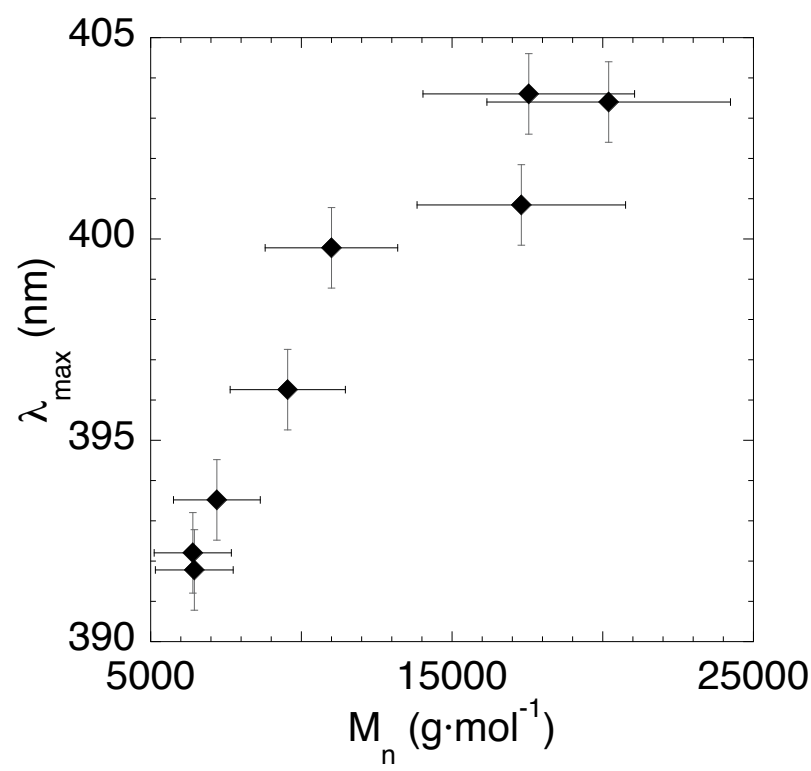

Figure 2. Variation with molecular weight of the peak position in the UV-visible absorption spectra of P3TMA in THF.

\section{Synthesis of poly(thiophene-3-sodium acetate)}

The saponification of the P3TMA was performed under milder conditions than those used in the work by Kim et al. ${ }^{27}$ We first solubilized the P3TMA powder in THF (2g/10 mL). Then 50 $\mathrm{mL}$ of concentrated $\mathrm{NaOH}$ solution $(7.5 \mathrm{M})$ was added. Although precipitation occurred, the particules formed were smaller than if the P3TMA powder was directly added to the $\mathrm{NaOH}$ solution. The solution was heated to $80^{\circ} \mathrm{C}$ for 1 hour under $\mathrm{Ar}$ atmosphere until the precipitate was no longer visible. Then THF was eliminated by heating at $90^{\circ} \mathrm{C}$ and allowing an Ar flux for a few minutes. The solution was kept at $90^{\circ} \mathrm{C}$ for 6 to 7 hours. Heating under Ar atmosphere was found critical to avoid polymer degradation under these harsh conditions. The solution was then cooled down and diluted by a factor 20 to 25 through the addition of distilled water. About $95 \%$ of the excess $\mathrm{NaOH}$ was neutralized by the addition of a concentrated $(2 \mathrm{M}) \mathrm{HCl}$ solution. The resulting homogeneous and bright-red colored solution was then dialyzed (Orange Scientific membranes, $3500 \mathrm{~g} / \mathrm{mol}$ cut-off) for 4 days 
until its conductivity fell below $5 \mu \mathrm{S}$. The dialysis was done first against distilled water with conductivity about $18 \mu \mathrm{S}$ and then against milli-Q water with conductivity below $3 \mu \mathrm{S}$. Dialysis in these conditions yields a partial acidification of the polyelectrolyte chains since poly(thiophene-3-acetic acid) is a weak polyacid. Therefore an aliquot of the solution was titrated forth and back with $\mathrm{NaOH}$ and $\mathrm{HCl}$ to determine the true neutralization degree of the polymer in the dialyzed solution (see below). The appropriate amount of $\mathrm{NaOH}$ was then added to recover fully neutralized P3TNaA chains that were isolated by freeze-drying. The water content of the final red-orange solid was determined by thermogravimetric analysis to be about $10 \%$ and was taken into account for the preparation of samples. The final product was stored in the dark, under argon, at room temperature.

\section{Characterization of the P3TNaA}

UV-visible spectroscopy. For more diluted solutions, the saponification could be followed in-situ by UV-visible absorption spectroscopy and we checked that $\lambda_{\max }$ and the related absorbance $\varepsilon_{\max }$ came to a level much sooner than assumed in ref. (27). The completion of the reaction under our experimental conditions was checked by NMR spectroscopy. For a solution with fully neutralized (see below) $\mathrm{P}_{3} \mathrm{TNaA}$ in $\mathrm{H}_{2} \mathrm{O}$, we found $\lambda_{\max }=435 \mathrm{~nm}$ and $\varepsilon_{\max }=6600 \mathrm{~cm}^{-1} \mathrm{M}^{-1}$.

NMR spectroscopy. The spectrum, measured in $\mathrm{D}_{2} \mathrm{O}$, reveals two broads peaks at peaks between 7.14 and $3.5 \mathrm{Ppm}$ corresponding to the aromatic protons and the methylene group of P3TNaA. On the other hand, the characteristic peaks of the protons of methoxy group were totally absent from the NMR spectrum of the polyelectrolyte in $D_{2} O$. This shows that the saponification was complete and yielded one ionizable group per monomer.

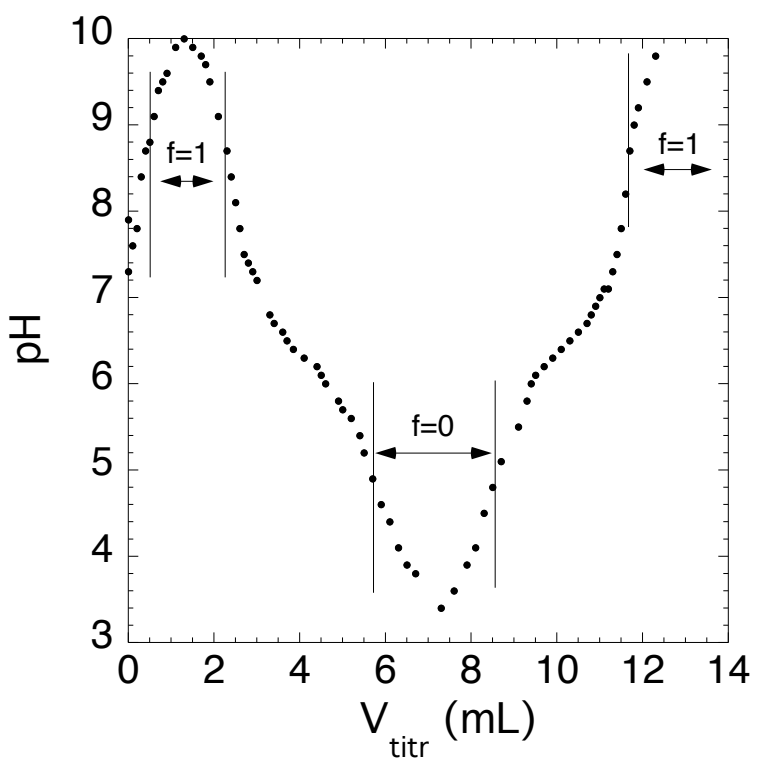

Figure 3. Titration curve of $\mathrm{P}_{3}$ TAA in $\mathrm{H}_{2} \mathrm{O}(C \approx 0.002 \mathrm{~mol} / \mathrm{L}): V_{\text {titr }}$ is the total volume of titrating 
solution, either $\mathrm{NaOH}$ (increasing $\mathrm{pH}$ )) or $\mathrm{HCl}$ (decreasing $\mathrm{pH}$ ). In the regions marked as $f=0$ and $f=1$, all the monomer units have been titrated and the $\mathrm{pH}$ evolution is linked to the addition of an excess of $\mathrm{NaOH}$ or $\mathrm{HCl}$.

Titration. Titration was performed on a $50 \mathrm{~mL}$ solution containing about $0.002 \mathrm{~mol} / \mathrm{L}$ of the partly acidified sample from the dialyzed solution at $\mathrm{T}=20^{\circ} \mathrm{C}$. One difficulty is that the polymer concentration is not known precisely. Therefore we first titrated the remaining acid functions with a $\mathrm{NaOH}$ solution $(0.02 \mathrm{M})$ until the $\mathrm{pH}$ evolution followed the one expected for a solution with increasing $\mathrm{NaOH}$ concentration. The solution was then back titrated with a $\mathrm{HCl}$ solution $(0.02 \mathrm{M})$ until the $\mathrm{pH}$ showed the behavior expected for a solution with increasing $\mathrm{HCl}$ concentration (see $\mathrm{Fig}$. 3). The concentration and neutralization degree of the chains in the dialyzed solution were then deduced from these two points and from the known molarities of the titrating solutions. A second titration by a $\mathrm{NaOH}$ addition confirmed the $\mathrm{PH}$ values at $\mathrm{f}=\mathrm{O}$ and $\mathrm{f}=1$ (Fig. 3). The uncertainties linked to the location of the inflexion points could be removed by the combined use of potentiometry with UVvisible absorption spectrocopy.

Field-Flow-Fractionation.

Field-Flow-Fractionation (FFF) coupled with light scattering was used to characterize the highest molecular weight fraction. The instrument is equipped with a pump (Agilent 1100 Series), an automated valve system (Eclipse2, Wyatt Tech.), a refractometer (Optilab rex, Wyatt Tech.) and a multi-angle light detection (Dawn Heleos, Wyatt Tech.): 50 to $100 \mu \mathrm{L}$ of a $2 \mathrm{mg} / \mathrm{mL}$ solution were injected in the flow cell equipped with a spacer of $490 \mu \mathrm{m}$ and a membrane with $5000 \mathrm{~g} / \mathrm{mol}$ cut-off. The direct solvant flux was $1 \mathrm{~mL} / \mathrm{min}$ and the cross-flow rate was varied between 4.5 and $1.0 \mathrm{~mL} / \mathrm{min}$. Finally we estimated $M_{w}=47000, M_{n}=20000, M_{w} / M_{n}=2.4$. Taking into account the slight increase of the repetitive unit mass (from $154 \mathrm{~g} / \mathrm{mol}$ to $162 \mathrm{~g} / \mathrm{mol}$ ) and considering the experimental errors in the difficult characterization of these samples by two different methods, these values are in good agreement with that of the native P3TMA chains. It can be noticed that these samples adsorb on aqueous SEC columns and that FFF in organic solvent was not available. Nevertheless we can exclude extensive chain degradation during the saponification process under our experimental conditions. In the following we will consider that the degree of polymerization DP of the chains is simply $D P=M_{n} / 162$ and we estimate the relative error on these values to be about 0.20. 